\title{
BMJ Open Study protocol for optimising glycaemic control in type 1 diabetes treated with multiple daily insulin injections: intermittently scanned continuous glucose monitoring, carbohydrate counting with automated bolus calculation, or both? A randomised controlled trial
}

\author{
Anna Lilja Secher (D) , ${ }^{1}$ Ulrik Pedersen-Bjergaard, ${ }^{2,3}$ Ole Lander Svendsen, ${ }^{4}$ \\ Birthe Gade-Rasmussen, ${ }^{5}$ Thomas P Almdal, ${ }^{6}$ Liv Dørflinger, ${ }^{7}$ Dorte Vistisen, ${ }^{1}$ \\ Kirsten Nørgaard ${ }^{1}$
}

To cite: Secher AL, PedersenBjergaard U, Svendsen OL, et al. Study protocol for optimising glycaemic control in type 1 diabetes treated with multiple daily insulin injections: intermittently scanned continuous glucose monitoring, carbohydrate counting with automated bolus calculation, or both? A randomised controlled trial. BMJ Open 2020;10:e036474. doi:10.1136/ bmjopen-2019-036474

- Prepublication history and additional material for this paper are available online. To view these files, please visit the journal online (http://dx.doi. org/10.1136/bmjopen-2019036474).

Received 17 December 2019 Revised 27 March 2020 Accepted 30 March 2020

\section{Check for updates}

(C) Author(s) (or their employer(s)) 2020. Re-use permitted under CC BY-NC. No commercial re-use. See rights and permissions. Published by BMJ.

For numbered affiliations see end of article.

\section{Correspondence to}

Dr Anna Lilja Secher; anna.elisabet.lilja.secher@ regionh.dk

\section{ABSTRACT}

Introduction There are beneficial effects of advanced carbohydrate counting with an automatic bolus calculator $(A B C)$ and intermittently scanned continuous glucose monitoring (isCGM) in persons with type 1 diabetes. We aim to compare the effects of isCGM, training in carbohydrate counting with $\mathrm{ABC}$ and the combination of the two concepts with standard care.

Methods and analysis A multi-centre randomised controlled trial with inclusion criteria: $\geq 18$ years, type 1 diabetes $\geq 1$ year, injection therapy, $\mathrm{HbA1c}>53 \mathrm{mmol} /$ mol, whereas daily use of carbohydrate counting and/ or CGM/isCGM wear are exclusion criteria. Inclusion was initiated in October 2018 and is ongoing. Eligible persons are randomised into four groups: standard care, $A B C$, isCGM or $\mathrm{ABC}+$ isCGM. Devices used are FreeStyle Libre Flash and smart phone diabetes application mySugr. Participants attend group courses according to treatment allocation with different educational contents. Participants are followed for 26 weeks with clinical visits and telephone consultations. At baseline and at study end, participants wear blinded CGM, have blood samples performed and fill in questionnaires on person-related outcomes, and at baseline also on personality traits and hypoglycaemia awareness. The primary outcome is the difference in time spent in normoglycaemia $(4-10 \mathrm{mmol} / \mathrm{L})$ at study end versus baseline between the isCGM group and the standard care group. Secondary outcomes will also be analysed. Results are expected in 2020.

Ethics and dissemination Regional Scientific Ethics Committee approval (H-17040573). Results will be sought disseminated at conferences and in high impact journals.

Trial registration number ClinicalTrial.gov registry (NCT03682237).

\section{Strengths and limitations of this study}

Despite its relevance for many persons with diabetes and their caregivers, the topic has not yet been rigorously examined to evaluate efficacy on glycaemic control.

- Robustly designed randomised controlled trial.

- Possible limitations may be difficulty with recruiting enough participants in order to reach power estimates, unbalanced withdrawal and basal insulin reductions which may affect the results.

\section{INTRODUCTION}

Insulin therapy in type 1 diabetes is generally adjusted according to food intake, physical activity and current blood glucose levels. It typically involves the combination of fast-acting insulin before meals and to correct hyperglycaemia, and long-acting insulin to control blood glucose overnight and in between meals, referred to as multiple daily injections (MDI). In order to obtain optimal glycaemic control, it requires dose adjustment based on the amount of carbohydrate ingested at each meal $^{1}$ and frequent self-monitoring of blood glucose (SMBG) measurements. Carbohydrate counting is done based on experience or by using either manual or automatic estimates. Several studies have shown improved HbAlc and treatment satisfaction as well as a tendency towards less hypoglycaemia in persons undergoing training in carbohydrate 
counting and manual bolus calculation compared with insulin dosing based on experience. ${ }^{1-3}$ In addition, the use of an automatic bolus calculator (ABC) rather than manual bolus calculation has been proved to reduce HbAlc and improve patient satisfaction, ${ }^{2}$ with unaffected hypoglycaemia rate. ${ }^{4}$ Even in a routine care setting, group courses of 4 hours in bolus calculation with $\mathrm{ABC}$ and follow-up consultations have shown a reduction in HbAlc with maintained effect 12 months post-course. ${ }^{5}$ Our experience is that many persons with type 1 diabetes have never been trained in carbohydrate counting or the algorithm for insulin dose calculation.

Compared with conventional SMBG, the use of continuous glucose monitoring (CGM) in MDI-treated persons reduces $\mathrm{HbA1c}$ and the time spent in mild hypoglycaemia and improves treatment satisfaction. ${ }^{6-8}$ A variation of realtime CGM is the intermittently scanned CGM (isCGM), where the first-generation readers are without glycaemic alerts, and the wearer scans the sensor to transfer glucose data. An early generation of isCGM has been found to decrease time spent in hypoglycaemia and increase treatment satisfaction and other person-related outcomes in people with near-optimal HbA1c at baseline. ${ }^{9}$ A number of observational studies have evaluated isCGM in terms of accuracy and change in $\mathrm{HbAlc},{ }^{10}$ with positive reports on wearer satisfaction. ${ }^{11}$ Two recent observational studies including 120 and 900 adults, respectively, mainly on MDI found improved HbAlc levels, however, with moderate increase in mild hypoglycaemic events, ${ }^{12}{ }^{13}$ but fewer diabetic ketoacidosis admissions. ${ }^{13}$ The most pronounced reduction in $\mathrm{HbAlc}$ was seen in those with higher $\mathrm{HbAlc}$ prior to isCGM use. ${ }^{13}$ To date, however, no randomised controlled trials exist examining the efficacy of isCGM to increase time in range in patients with suboptimal HbAlc.

It also remains unknown which of the two concepts, bolus calculation with $\mathrm{ABC}$ or the use of isCGM, is superior and whether there is an additive effect of the two combined. Furthermore, there are no studies on how to structure training and optimise treatment in patients doing bolus calculation with the concomitant use of isCGM.

In the current study, the primary aim is to compare the effect of isCGM with standard care (SMBG) on glycaemic control. The secondary aim is to compare the effect of the combination of training in carbohydrate counting with $\mathrm{ABC}$ and the use of isCGM with standard care. We also aim to assess whether isCGM use can outperform training in carbohydrate counting with ABC.

\section{METHODS AND ANALYSIS \\ Participants}

The ABC Flash Study is a randomised controlled, openlabel, four-arm parallel trial carried out at five sites in The Capital Region of Denmark: Steno Diabetes Center Copenhagen, Rigshospitalet, Amager and Hvidovre Hospital, Nordsjællands Hospital Hillerød, and Bispebjerg and Frederiksberg Hospital. Inclusion criteria are age $\geq 18$ years, type 1 diabetes duration $\geq 1$ year, $\mathrm{HbA1c}>53$ $\mathrm{mmol} / \mathrm{mol}$ and the use of MDI therapy with basal insulin accounting for $\geq 30 \%$ of the total daily insulin dose. Exclusion criteria are daily use of carbohydrate counting or CGM, use of neutral protamine Hagedorn (NPH) insulin, pregnancy, breastfeeding, gastroparesis, severe diabetes complications (ie, proliferative retinopathy, myocardial infarction within the last 6 months), other medical or psychological conditions judged unsuitable for study participation, participation in other diabetes-related clinical research, the use of other drugs than insulin affecting glucose metabolism, or the inability to understand the individual information and to give informed consent. All persons screened for participation fill out the ABC Flash Study patient consent form (online supplementary file $1)$. Hence, participants are adults with type 1 diabetes and suboptimal glycaemic control, treated with basal and bolus insulin and attending an outpatient diabetes clinic in the Capital Region of Denmark. Screening for inclusion was initiated on 1 October 2018 and is planned to continue until 31 March 2020.

\section{Intervention}

Automatic bolus calculator

The ABC system used for this trial is the CE-marked mobile telephone diabetes application mySugr available for Android and iPhone. It is a digital logbook designed to support persons with diabetes in their selfmanagement. It consists of an insulin bolus calculator based on the following settings that can be individually adjusted for every half an hour lap though the 24 hours: carbohydrate ratio, insulin sensitivity, target glucose value and insulin duration time. For every meal, the user enters current glucose value and estimated carbohydrate intake and receives a suggestion on insulin bolus dosage. In case of correction of hyperglycaemia, the user enters current glucose value and receives a suggestion on corrective insulin dosage. Data are stored for 3 months.

\section{Intermittently scanned CGM}

The isCGM device used in this trial is the FreeStyle Libre Flash CGM system (Abbott Diabetes Care, Alameda, California, USA). It consists of a 14-day disc sensor to be worn on the upper arm, a reader and/or a mobile phone application to scan and display data. The sensor measures and continuously stores interstitial glucose concentrations every 15 min ( 800 glucose readings/ 24 hours). The sensor is inserted into the upper arm skin and placed by a thin needle, which is immediately retracted, leaving a thin $6 \mathrm{~mm}$ long plastic glucose recorder in the skin. To obtain a current glucose value, the wearer scans the sensor with either the reader device or a mobile phone application (FreeStyle Libre Link available for Android and iPhone, Abbott Diabetes Care) producing real-time data. The reader devices used for this study were updated mid-2019. ${ }^{14}$ The application was available in Denmark for Android and iPhone from mid-2019. ${ }^{15}$ The system is CE-marked and accurate enough for insulin dosing 
except during fluctuant and low glucose levels. Wearers do not need to calibrate the system but to avoid loss of data, the sensor must be scanned every 8 hours. Scanned glucose data are presented to the wearer on the display as numerical values including glucose trends based on automatically stored data. Both intermittently scanned as well as continuously stored glucose data are displayed as graphs and logbooks and are available as numerical values after download from the reader device/mobile phone to a computer by the software program Diasend (Glooko, USA).

\section{Assignment of interventions}

Persons eligible for inclusion are randomised 1:1:1:1 by drawing a sealed and opaque randomisation envelope at the screening visit. The envelopes were centrally prepared by a person without relation to the specific trial. The main site was assigned to screen 72 participants, while the four remaining sites were each assigned to screen 32 participants. There is equal distribution of group assignments at each site (18 at the main site; 8 at each of the other four sites). The envelope is left unopened until after the initial blinded CGM period, where the given intervention is revealed: A (standard care), B (ABC), C (isCGM) or D (ABC and isCGM). In case of drop-out, the participant's allocation is not to be replaced in the remaining randomisation envelopes.

\section{Courses for participants}

Four types of group courses for participants are included in the trial (table 1). Each course includes one to three educational elements according to group allocation. Course length (4-4.5 hours) and group size (4-6 participants) are rather similar between the groups. The educational elements are:

1. General diabetes: training in general diabetes health issues, how to do experience-based dosing, how to handle sick days, exercise and so on in general terms.
2. ABC: theoretic and practical training in carbohydrate counting and bolus calculation. ${ }^{5}$

3. mySugr diabetes application: the application is downloaded on the participants personal smartphone. The insulin to carbohydrate ratio (the amount of carbohydrates needed to match the glucose lowering effect of 1 unit of subcutaneously injected rapid-acting insulin) and the insulin sensitivity (the decrease in blood glucose in mmol/L caused by 1 unit of subcutaneously injected rapid-acting insulin) are empirically estimated for each participant using the 500-rule and the 100rule, respectively. ${ }^{16}$ The insulin duration time is set at 4 hours. The target glucose value is in general set at 6 $\mathrm{mmol} / \mathrm{L}$ during daytime and $7 \mathrm{mmol} / \mathrm{L}$ during night time.

4. FreeStyle Libre Flash CGM: participants are instructed to use the system according to our local guideline and those in group $\mathrm{D}$ are also instructed in how to incorporate isCGM trend arrows to adjust the mySugr application settings.

All the above-mentioned educational concepts are also practiced during individual consultations throughout the 26-week trial period.

\section{Training of study personnel}

The healthcare professionals at the different study sites have been educated in the protocol and different course content and treatment modalities, how to conduct courses and study visits on two separate days before inclusion was initiated. Teachers on these study personnel training sessions were an endocrinologist $(\mathrm{KN})$, a diabetes nurse and a dietician from the investigator group with substantial experience in the field.

\section{Diabetes care}

In line with national guidelines, ${ }^{17}$ the overall aim in term of diabetes management is to obtain fasting and pre-prandial glucose values of 4-6 mmol/L, post-prandial glucose values

Table 1 Overview of glucose measurement methods, decision on insulin bolus and educational element in course according to group allocation

\begin{tabular}{llll}
\hline $\begin{array}{l}\text { Intervention } \\
\text { group }\end{array}$ & $\begin{array}{l}\text { Glucose } \\
\text { measurement } \\
\text { method }\end{array}$ & Decision on insulin bolus & Educational element in course \\
\hline A & SMBG & Experience-based & General diabetes \\
B & SMBG & $\begin{array}{l}\text { Carbohydrate counting with } \\
\text { automatic bolus calculator }\end{array}$ & $\begin{array}{l}\text { Training in carbohydrate counting } \\
\text { Training in the use of the application mySugr }\end{array}$ \\
C & isCGM & Experience based & $\begin{array}{l}\text { General diabetes } \\
\text { Training in FreeStyle Libre Flash use }\end{array}$ \\
& isCGM & $\begin{array}{l}\text { Carbohydrate counting with } \\
\text { automatic bolus calculator }\end{array}$ & $\begin{array}{l}\text { Training in carbohydrate counting } \\
\text { Training in the use of the application mySugr } \\
\text { Training in FreeStyle Libre Flash use and how to incorporate } \\
\text { glucose trend arrows to adjust the mySugr application } \\
\text { settings }\end{array}$ \\
\hline
\end{tabular}

SMBG, self-monitoring of blood glucose; isCGM, intermittently scanned continuous glucose monitoring. 
lower than $10 \mathrm{mmol} / \mathrm{L}$, to avoid any glucose values lower than $3 \mathrm{mmol} / \mathrm{L}$ and keep the number of glucose values below $4 \mathrm{mmol} / \mathrm{L}$ at an absolute minimum. At baseline and up to the last study visit, all participants fill in work sheets for daily reporting of basal insulin units, insulin boluses and SMBG values during the 2 weeks of blinded CGM wear to adjust insulin therapy. Moreover, participants receive a hypoglycaemia diary to fill in symptoms of hypoglycaemia and/or glucose values below $3.9 \mathrm{mmol} / \mathrm{L}$, throughout the trial which is also used for insulin adjustments.

Participants in groups A (standard care) and B (ABC) are encouraged to measure SMBG at least four times daily with their personally preferred glucose metre. Participants in groups $\mathrm{C}$ (isCGM) and $\mathrm{D}$ (isCGM and $\mathrm{ABC}$ ) are instructed to use the isCGM system according to a local guideline based on manufacturer's guideline ${ }^{18}$ and in line with recent publications on the topic. ${ }^{19-21}$ During both study visits and telephone consultations (see below) study personnel titrate insulin doses are based on the different types of glucose values provided and other clinical information that is, hypoglycaemic events, planned physical activity and so on. For participants in groups $\mathrm{B}$ and $\mathrm{D}$, both the basal insulin dose and the $\mathrm{ABC}$ settings (primarily carbohydrate ratio and insulin sensitivity) are evaluated and, if needed, adjusted according to a local guideline based on previous publications and clinical experience. ${ }^{22}$

\section{Outcomes}

The primary outcome is the difference in change from baseline to end of study between groups C (isCGM) and A (standard care) in time spent in normoglycaemia (defined as glucose of $4-10 \mathrm{mmol} / \mathrm{L}$, minutes $/ 24$ hours, obtained by blinded $\mathrm{CGM}^{23}$ ).

Secondary outcomes are: difference in change from baseline to end of study between groups in $\mathrm{HbAlc}$ ( $\mathrm{mmol} /$ mol), difference between groups in severe hypoglycaemia occurrence during the study period (defined as an event requiring assistance of another person, plasma glucose concentrations may not be available during an event, but neurological recovery following the return of plasma glucose to normal, number of events during study period), difference between groups in symptomatic and confirmed hypoglycaemia occurrence during the study period (defined as glucose (SMBG or isCGM) $<3 \mathrm{mmol} / \mathrm{L}$, number of events per week), difference in change between baseline and end of study between groups in diabetes distress, diabetes treatment satisfaction, diabetes empowerment, diabetes quality of life, time spent in hypoglycaemia (blinded CGM glucose $<3 \mathrm{mmol} / \mathrm{L},<4 \mathrm{mmol} / \mathrm{L}$, minutes/ day) and hyperglycaemia ( $>10 \mathrm{mmol} / \mathrm{L}$, minutes/day), glycaemic variability $(\mathrm{SD})$, total insulin dose (recorded as a mean of 2 weeks during blinded CGM (insulin units (IU) / day/ $\mathrm{kg}$ ), total basal insulin dose (IU/day/ $\mathrm{kg}$ ), insulin boluses (number/24 hours), body weight $(\mathrm{kg})$ and urinary albumin/excretion rate ( $\mathrm{mg} / 24$ hours), and last the association between personality traits evaluated by questionnaire at baseline with any other outcome measures in the different groups.

\section{Data collection}

\section{Blinded CGM}

After screening for inclusion (before opening the randomisation envelope), and at study end, all participants are asked to wear a blinded (non-real-time) CGM (The FreeStyle Libre Professional CGM system, Abbott Diabetes Care, Oxon, UK) to obtain glucose data. The sensor is inserted by a healthcare professional (see above for details on the isCGM). While wearing the sensor, users do not need to enter any fingerstick data or carry around a receiver, since the device collects all glucose data automatically. Following 2 weeks of sensor wear, data are downloaded in office by a reader device and a manufacturer-provided computer software program (LibreLink, Abbott Diabetes Care). These data are collected between screening visit and course participation (baseline data) and the two last study visits (final data), respectively.

\section{Glucose and $A B C$ data}

Glucose (SMBG or isCGM) are downloaded to a computer (software Diasend, Glooko, USA) and data from the ABC are sent by email from the user to the project healthcare professional at study visits 2, 4 and 6 . The average number of symptomatic mild hypoglycaemic episodes per week and any severe hypoglycaemic episode are consecutively recorded throughout the trial.

\section{Questionnaires}

At screening and last study visit, all participants fill in the following validated questionnaires: Problem Areas in Diabetes Questionnaire (PAID), Diabetes Treatment Satisfaction Questionnaire (DTSQs at baseline and DTSQs and DTSQc at last visit), ${ }^{24}$ Diabetes Empowerment Scale (DES short form) and Audit of Diabetes Dependent Quality of Life (ADDQoL-19). At screening, they also fill in the questionnaire Neuroticism Extraversion Openness Agreeableness Conscientiousness Five-Factor Invertory-3 (NEO-FFI-3) and hypo-awareness assessment. ${ }^{25-28}$

\section{General health, blood and urine sampling and body weight}

A general health/safety assessment, including full objective examination (cardiac and pulmonal auscultation, blood pressure measurement, inspection of insulin injection sites and so on) and information on history of severe hypoglycaemia is performed at screening. At both screening and last visit, all participants have blood and urine samples taken, as well as body weight measured $(\mathrm{kg})$, which is done using the same scale every time. HbAlc $(\mathrm{mmol} / \mathrm{mol})$ is measured at screening, midway through the study, and again at last visit.

\section{Study visits and telephone consultations}

There are in total five clinical follow-up study visits (visits 2-6) at 2, 4, 12, 24 and 26 weeks planned during the 26 weeks trial participation (see figure 1). Participants in groups A (standard care) and C (isCGM) have appointments with the project nurse, whereas participants in groups $\mathrm{B}(\mathrm{ABC})$ and $\mathrm{D}$ (isCGM and $\mathrm{ABC}$ ) have joint appointments with both the project nurse and the project dietician. All participants have appointments 


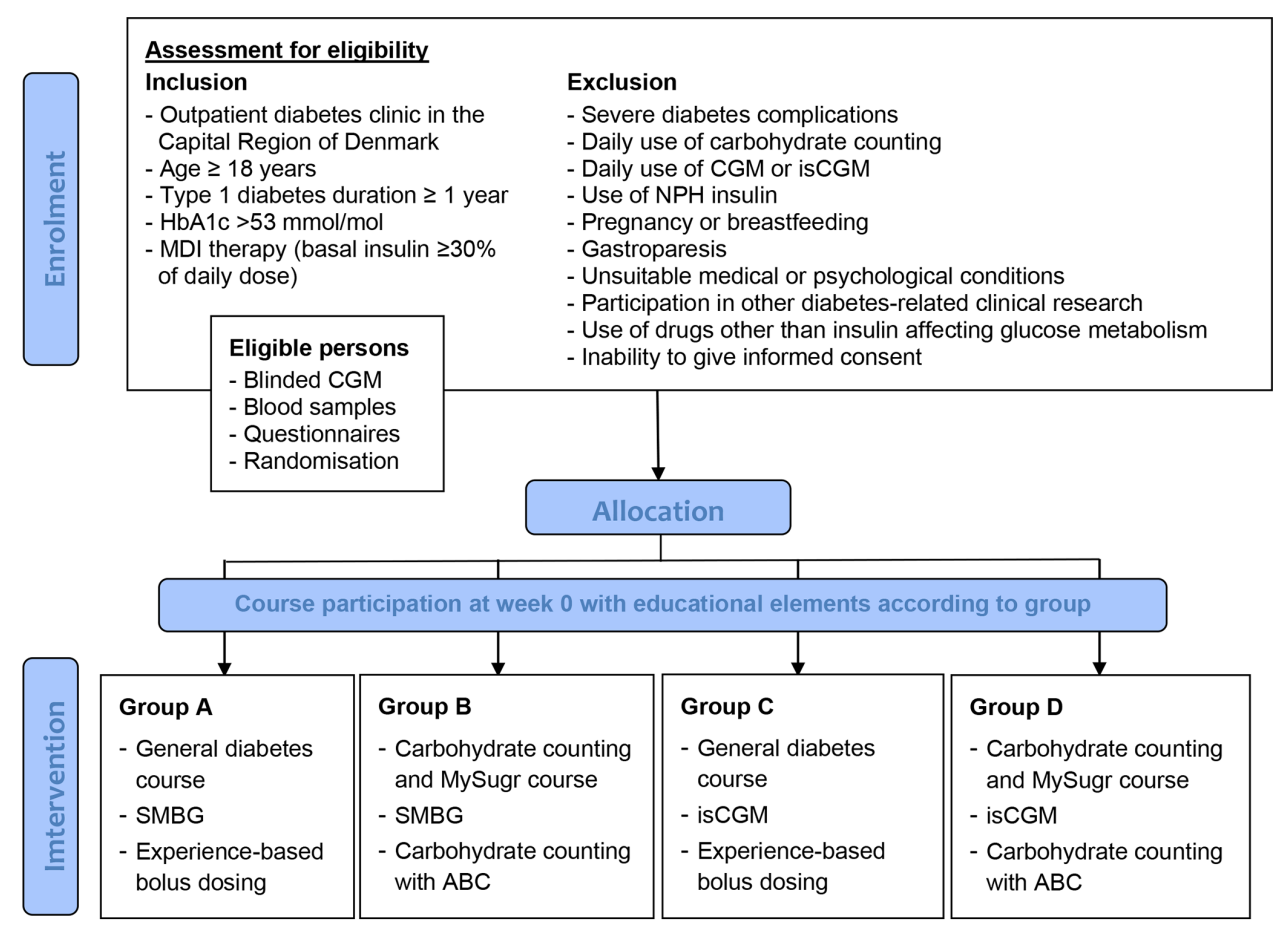

Study visits - Clinical visits at 2, 4, 12, 24 and 26 weeks and telephone visits at 0,8 and 17 weeks. Study end - Blinded CGM, blood samples, questionnaires.

Primary outcome
The difference in change from baseline to end of study between groups $\mathrm{C}$ (isCGM) and A (standard care)
in time spent in normoglycaemia (4-10 mmol/l).
Secondary outcomes will also be analysed.

Figure 1 Flow chart of participants throughout the trial.

with the project physician at first visit (screening) and midways throughout participation (visit 4). Visit 3 is not mandatory but proposed to participants in need of extra support or renewed teaching in the allocated intervention. In between the clinical study visits, there are three telephone consultations ${ }^{1-3}$ at 0,8 and 17 weeks, where the project nurse contacts the participants.

\section{Data management}

The data management is performed in accordance with the General Data Protection Regulation. Data are consecutively collected and stored in a browser-based software and workflow database (REDCap). The database is password protected and only the local and primary investigators have access to the data.

\section{Data analysis}

A sample size of 160 (40 per group) was calculated to have $80 \%$ power to detect a difference in mean time in target glycaemic range $(4-10 \mathrm{mmol} / \mathrm{L})$ between treatment groups $\mathrm{A}$ and $\mathrm{C}$ of $75 \mathrm{~min} /$ day with an $\mathrm{SD}$ of $120 \mathrm{~min}$, and a two-sided $\alpha$-level of 0.05 . Sample size is increased to 180 (45 per group) to account for a potential drop out of approximately $10 \%$.
Changes in primary and secondary outcomes over the intervention period and effects of the treatments will be modelled by linear mixed-effects models with a patientspecific random intercept to account for the correlation of repeated measurements within patients and a random intercept for centre to account for the clustering effect of study centre. The exact times of measurements will be used. All analyses will be performed as an intention to treat analysis. Statistical significance will be inferred at a two-tailed $\mathrm{p}<0.05$. The $\mathrm{p}$-values for secondary outcomes will be corrected by the Benjamini-Hochberg method for multiple comparisons. ${ }^{29}$

\section{Public and patient involvement}

Persons with type 1 diabetes were not involved in the initial phases of the study. Already recruited participants, however, are asked to assess the burden of the intervention and time required to participate in the research. This has resulted in a online supplementary file 1 document that is presented to possible participants to give a realistic picture of time spent on participation over time. Persons with type 1 diabetes will be sought to be involved in the dissemination plan of the results by, for example, 
commenting on written information with regards to language and form.

\section{Ethics and dissemination}

The study is carried out in accordance with the Helsinki Declaration after approval by the Regional Scientific Ethics Committee (H-17040573).

Until now, an early generation isCGM has been found to improve time spent in hypoglycaemia, treatment satisfaction and other person-related outcomes in people with near-optimal HbA1c. ${ }^{9}$ A number of observational studies have evaluated isCGM in broader populations with regards to change in HbAlc and accuracy and satisfaction among wearers. ${ }^{10-13}$ To date, however, no randomised controlled trials have been performed to examine the efficacy of isCGM to increase time in range in patients with suboptimal HbAlc. In addition, carbohydrate counting with $\mathrm{ABC}$ has been proved to improve HbAlc and patient satisfaction ${ }^{2}$ and nutritional therapy has an integral role in diabetes management. ${ }^{30}$ Which one of the two treatment concepts that is superior to the other, and whether there is an additive effect of the two combined, has to our knowledge not previously been evaluated. Most important, however, no randomised controlled trials have been performed to examine the efficacy of isCGM to increase time in range in patients with suboptimal HbA1c. A possible limitation may be difficulty with recruiting enough participants in order to reach power estimates. Unbalanced withdrawal may be a risk, as we believe that the demands are higher on participants allocated to one of the two carbohydrate counting groups. Furthermore, as isCGM is not fully reimbursed in Denmark, some participants may sign up for the study with the hope of being randomised to isCGM. Basal insulin reductions may also play a role in achieving glycaemic aims.

The investigators expect that the current study results will help guiding persons with type 1 diabetes treated with MDI and their healthcare professionals in choosing evidencebased methods for optimal glycaemic control. We believe that the possible risks and side effects among participants are outweighed by the potential benefits from the conduct of this study. The overall individual risk of side effects is expected to be modest and is mainly related to the time spent on learning how to count carbohydrates and use the mySugr application and/or using the isCGM system. With regards to all other planned study procedures, the risk of complications or adverse events is negligible and outweighed by the possible beneficial effects of conducting the study. The occurrence of any adverse events will be assessed at every visit and telephone contact during the study period. All participants are covered by the mandatory individual insurance at each local hospital in The Capital Region of Denmark. Blood samples are analysed directly after sampling without the establishment of a biobank. If the study is prematurely terminated, the investigators will promptly inform the Regional Scientific Ethics Committee and the participants to assure appropriate therapy and follow-up.
The study results are expected to be disseminated at international and national diabetes conferences and meetings and published in international journals with considerable impact. All participants who at first study visit expressed an interest in the results will receive a short version of the main findings expressed in lay terms and will be invited to a short oral presentation at the main study site. The results will also be sought presented to the Danish Diabetes Association and communicated to the public by a press release.

\section{Author affiliations}

${ }^{1}$ Clinical Research, Steno Diabetes Center Copenhagen, Gentofte, Denmark ${ }^{2}$ Endocrine Section, Department of Endocrinology and Nephrology, Nordsjællands Hospital, Hillerod, Denmark

${ }^{3}$ Faculty of Health and Medical Sciences, University of Copenhagen, Kobenhavn, Denmark

${ }^{4}$ Department of Endocrinology I, Bispebjerg Hospital, Kobenhavn, Denmark

${ }^{5}$ Department of Endocrinology, Hvidovre Hospital, Hvidovre, Denmark

${ }^{6}$ Department of Endocrinology, Rigshospitalet, Kobenhavn, Denmark

${ }^{7}$ Administration, Steno Diabetes Center Copenhagen, Gentofte, Denmark

Contributors ALS wrote the first draft of the manuscript. Critical revision of the manuscript: UP-B, OLS, BG-R, TPA, LD, DV and KN. KN is the primary investigator of the study. The study concept, trial design and study protocol: ALS, UP-B, OLS, BG-R, TPA, LD and DV.

Funding The study is investigator-initiated and investigator-driven, financed by The Capital Region of Denmark. None of the investigators have personal financial interest in the conduct or the outcome of the project.

Competing interests KN is a shareholder of Novo Nordisk; has received research support from Novo Nordisk, Roche Diagnostics, Dexcom and Zealand Pharma; has received lecture fees from Medtronic, Roche Diagnostics, Rubin Medical, Sanofi, Zealand Pharma, Novo Nordisk and Dexcom and has served on advisory panels for Medtronic, Abbott and Novo Nordisk. UP-B has served on advisory boards for AstraZeneca, Bristol-Myers Squibb, Sanofi-Aventis, Novo Nordisk and Zealand Pharma and has received lecture fees from AstraZeneca, Bristol-Myers Squibb, Sanofi-Aventis and Novo Nordisk. TPA holds stocks in Novo Nordisk. ALS, OLS, BG$\mathrm{R}, \mathrm{LD}$ and DV do not have any competing interests.

Patient and public involvement Patients and/or the public were not involved in the design, or conduct, or reporting, or dissemination plans of this research.

Patient consent for publication Not required.

Provenance and peer review Not commissioned; externally peer reviewed.

Open access This is an open access article distributed in accordance with the Creative Commons Attribution Non Commercial (CC BY-NC 4.0) license, which permits others to distribute, remix, adapt, build upon this work non-commercially, and license their derivative works on different terms, provided the original work is properly cited, appropriate credit is given, any changes made indicated, and the use is non-commercial. See: http://creativecommons.org/licenses/by-nc/4.0/.

ORCID iD

Anna Lilja Secher http://orcid.org/0000-0003-2247-6213

\section{REFERENCES}

1 Schmidt S, Schelde B, Nørgaard K. Effects of advanced carbohydrate counting in patients with type 1 diabetes: a systematic review. Diabet Med 2014;31:886-96.

2 Schmidt S, Meldgaard M, Serifovski N, et al. Use of an automated bolus calculator in MDI-treated type 1 diabetes: the BolusCal study, a randomized controlled pilot study. Diabetes Care 2012;35:984-90.

3 Bell KJ, Barclay AW, Petocz P, et al. Efficacy of carbohydrate counting in type 1 diabetes: a systematic review and meta-analysis. Lancet Diabetes Endocrinol 2014;2:133-40.

4 Hommel E, Schmidt S, Vistisen D, et al. Effects of advanced carbohydrate counting guided by an automated bolus calculator in type 1 diabetes mellitus (StenoABC): a 12-month, randomized clinical trial. Diabet Med 2017;34:708-15.

5 Meldgaard M, Damm-Frydenberg C, Vesth U, et al. Use of advanced carbohydrate counting and an automated bolus calculator in clinical 
practice: the BolusCal ${ }^{\circledR}$ training concept. International Diabetes Nursing 2015:12:8-13.

6 Beck RW, Riddlesworth T, Ruedy K, et al. Effect of continuous glucose monitoring on glycemic control in adults with type 1 diabetes using insulin injections: the diamond randomized clinical trial. JAMA 2017;317:19975:371

7 Lind M, Polonsky W, Hirsch IB, et al. Continuous glucose monitoring vs conventional therapy for glycemic control in adults with type 1 diabetes treated with multiple daily insulin injections: the gold randomized clinical trial. JAMA 2017;317:19976:379.

8 Beck RW, Bergenstal RM, Laffel LM, et al. Advances in technology for management of type 1 diabetes. Lancet 2019;394:1265-73.

9 Bolinder J, Antuna R, Geelhoed-Duijvestijn P, et al. Novel glucosesensing technology and hypoglycaemia in type 1 diabetes: a multicentre, non-masked, randomised controlled trial. Lancet 2016;388:2254-63.

10 Leelarathna L, Wilmot EG. Flash forward: a review of flash glucose monitoring. Diabet Med 2018;35:472-82.

11 Ólafsdóttir AF, Attvall S, Sandgren U, et al. A clinical trial of the accuracy and treatment experience of the flash glucose monitor FreeStyle Libre in adults with type 1 diabetes. Diabetes Technol Ther 2017;19:164-72.

12 Paris I, Henry C, Pirard F, et al. The new FreeStyle libre flash glucose monitoring system improves the glycaemic control in a cohort of people with type 1 diabetes followed in real-life conditions over a period of one year. Endocrinol Diabetes Metab 2018;1:e00023.

13 Tyndall V, Stimson RH, Zammitt NN, et al. Marked improvement in $\mathrm{HbA}_{1 \mathrm{c}}$ following commencement of flash glucose monitoring in people with type 1 diabetes. Diabetologia 2019;62:1349-56.

14 FreeStyle Libre. Available: https://www.freestyle.abbott/uk/en/readerupdate/index.html

15 FreeStyle Libre. Available: https://www.freestyle.abbott/ie/en/ librelink/index.html

16 Walsh P, Roberts R. Pumping insulin. San Diego, CA: Torrey Pines Press, 2006.

17 Danish Endocrine Society. Available: http://www.endocrinology.dk/ index.php/1-diabetes-mellitus/3-type-1-diabetes-mellitus

18 FreeStyle Libre. Available: https://www.freestylelibre.co.uk/libre/ discover/using-your-meter.htm
19 Bianchi C, Aragona M, Rodia C, et al. Freestyle Libre trend arrows for the management of adults with insulin-treated diabetes: a practical approach. J Diabetes Complications 2019;33:6-12.

20 Kudva YC, Ahmann AJ, Bergenstal RM, et al. Approach to using trend Arrows in the FreeStyle Libre flash glucose monitoring systems in adults. $J$ Endocr Soc 2018;2:1320-37.

21 Borot S, Benhamou PY, Atlan C, et al. Practical implementation, education and interpretation guidelines for continuous glucose monitoring: a French position statement. Diabetes Metab 2018;44:61-72.

22 Schmidt S, Nørgaard K. Bolus calculators. J Diabetes Sci Technol 2014;8:1035-41.

23 Battelino T, Danne T, Bergenstal RM, et al. Clinical targets for continuous glucose monitoring data interpretation: recommendations from the International consensus on time in range. Diabetes Care 2019;42:1593-603

24 Bradley C, Plowright R, Stewart J, et al. The diabetes treatment satisfaction questionnaire change version (DTSQc) evaluated in insulin glargine trials shows greater responsiveness to improvements than the original DTSQ. Health Qual Life Outcomes 2007;5:57.

25 Gold AE, MacLeod KM, Frier BM. Frequency of severe hypoglycemia in patients with type I diabetes with impaired awareness of hypoglycemia. Diabetes Care 1994;17:697-703.

26 Clarke WL, Cox DJ, Gonder-Frederick LA, et al. Reduced awareness of hypoglycemia in adults with IDDM. A prospective study of hypoglycemic frequency and associated symptoms. Diabetes Care 1995; 18:517-22.

27 Pedersen-Bjergaard U, Pramming S, Thorsteinsson B. Recall of severe hypoglycaemia and self-estimated state of awareness in type 1 diabetes. Diabetes Metab Res Rev 2003;19:232-40.

28 Høi-Hansen T, Pedersen-Bjergaard U, Thorsteinsson B. Classification of hypoglycemia awareness in people with type 1 diabetes in clinical practice. J Diabetes Complications 2010;24:392-7.

29 Benjamini Y, Hochberg Y. Controlling the false discovery rate: a practical and powerful approach to multiple testing. Journal of the Royal Statistical Society: Series B 1995;57:289-300.

30 American Diabetes Association. 4. Lifestyle Management: Standards of Medical Care in Diabetes-2018. Diabetes Care 2018:41:S38-50. 